Dom. Cien., ISSN: 2477-8818

Vol. 5, núm.1., ene, 2019, pp. 722-735

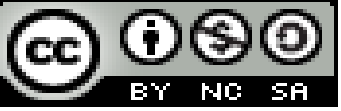

Ciencias de la salud

\title{
Percepción del climaterio en pacientes del centro de salud materno infantil "Francisco Jácome", plan de prevención
}

\section{Climaterio perception in patients of the center of maternal childhood health "Francisco Jácome", prevention plan \\ Percepção de climaterio em pacientes do centro de saúde de infância maternal "Francisco Jácome", plano de prevenção}

\author{
Alicia E. Requena-Vera \\ alicia.reque@hotmail.com \\ Beatriz M. Moran-Rivas ${ }^{\text {II }}$ \\ beatriz.moran-1@gamil.com \\ Jorge H. Labanda Casquete \\ III \\ jorge.labanda@gmail.com
}

Recibido: 27 de noviembre de 2018 * Corregido: 18 de diciembre de $2018 *$ Aceptado: 20 de diciembre de 2018

I. Magister en Gerencia Clínica en Salud Sexual y Reproductiva; Diplomado en Docencia Superior; Obstetriz; Docente de la Universidad de Guayaquil, Guayaquil, Ecuador.

II. Magister en Gerencia Clínica en Salud Sexual y Reproductiva; Diplomado en Docencia Superior; Obstetriz; Docente de la Universidad de Guayaqui, Guayaquil, Ecuador.

III. Magister en Gerencia Clínica en Salud Sexual y Reproductiva; Diploma Superior en Diseño Curricular por Competencias; Doctor en Medicina y Cirugía; Médico; Obstetra; Docente de la Universidad de Guayaquil, Guayaquil, Ecuador. 


\title{
Resumen
}

El climaterio es una etapa natural en la vida de la mujer, es la transición de la vida reproductiva a la no reproductiva, es un periodo con una duración de 10-15 años en el que se van a producir profundos cambios en el organismo a nivel físico, psicológico afectivo y la causa más importante es el cese de la función ovárica. El campo de investigación es la de percepción de los síntomas del síndrome climatérico en las mujeres de 40 a 65 años. Y La finalidad de este estudio es elaborar un plan preventivo para evitar complicaciones del síndrome climaterio a las usuarias del Centro Materno Infantil Francisco Jácome. Esta investigación es de tipo cualitativo, descriptivo, exploratorio, para realizar este estudio se establecen las categorías: educativa, psicológica, biológicas y cultural, se utilizaron encuestas a las usuarias con preguntas abiertas y cerradas que nos permitió abordar y conocer esta realidad, la mayoría de las mujeres estaban en la posmenopausia y la edad promedio de su última menstruación fue a los 48 años, se identificó que si hay deficiencia en la percepción del síndrome climatérico. Dentro de la sintomatología la mayoría de las usuarias presentan trastornos como los sofocos, sudoración, irritabilidad, depresión que afectan mayormente sus actividades diarias los trastornos de la esfera urogenital con sequedad vaginal y dispareunia fueron las más frecuentes. Por lo tanto, la propuesta está orientada a mejorar la percepción del síndrome climatérico y prevenir sus complicaciones.

Palabras claves: Percepción; Climaterio; Menopausia; Reproductiva; Mujeres.

\begin{abstract}
The climacteric is a natural stage in the life of the woman, it is the transition from the reproductive to the non-reproductive life, it is a period with a duration of 10-15 years in which profound changes in the organism are going to take place. physical, psychological affective and the most important cause is the cessation of ovarian function. The field of research is the perception of the symptoms of the climacteric syndrome in women aged 40 to 65 years. And The purpose of this study is to elaborate a preventive plan to avoid complications of the climacteric syndrome to the users of the Centro Materno Infantil Francisco Jácome. This research is qualitative, descriptive, exploratory, in order to carry out this study the categories are established: educational, psychological, biological and cultural, user surveys were used with open and closed questions that allowed us to approach and know this reality, the majority of the women were in the postmenopause and the average age of their
\end{abstract}


last menstruation was at 48 years, it was identified that if there is deficiency in the perception of the climacteric syndrome. Within the symptoms most users present disorders such as hot flashes, sweating, irritability, depression that mostly affect their daily activities disorders of the urogenital area with vaginal dryness and dyspareunia were the most frequent. Therefore, the proposal is aimed at improving the perception of the climacteric syndrome and preventing its complications.

Key words: Perception; Climacteric; Menopause; Reproductive Women.

\section{Resumo}

O climatério é uma fase natural na vida da mulher, é a transição da vida reprodutiva de nãoreprodução, é um período com uma duração de 10-15 anos em que irá produzir alterações profundas no nível organismo físico, psicológico afetivo e a causa mais importante é a cessação da função ovariana. O campo de pesquisa é a percepção dos sintomas da síndrome do climatério em mulheres de 40 a 65 anos. E o objetivo deste estudo é elaborar um plano preventivo para evitar complicações da síndrome do climatério para os usuários do Centro Materno Infantil Francisco Jácome. Esta pesquisa é qualitativa, descritiva e exploratória, por este categorias de estudo estão definidas: inquéritos pedagógicos, psicológicos, biológicos e culturais para os usuários com perguntas abertas foram utilizados e fechado que nos permitiu abordar e atender a essa realidade, a maioria as mulheres eram pós-menopausa ea idade média do seu último período foi aos 48 anos, foi identificado que, se há deficiência na percepção da síndrome climatérica. Entre os sintomas a maioria dos usuários têm distúrbios, tais como afrontamentos, sudação, irritabilidade, depressão que afetam principalmente os distúrbios atividades diárias esfera urogenital com a secura vaginal e dispareunia foram os mais frequentes. Portanto, a proposta visa melhorar a percepção da síndrome do climatério e prevenir suas complicações.

Palavras chave: Percepção Climatério; Menopausa; Reprodutiva Mulheres.

\section{Introducción.}

La palabra climaterio proviene del griego "Climacter" (peldaños o escalones) el climaterio es la etapa de transición que ocurre entre la madurez reproductiva a la no reproductiva, con la pérdida de la función ovárica el cual se caracteriza por una deficiencia de estrógenos, con los cambios o modificaciones biológicas, 
psicológicas, que conlleva este déficit hormonal, a su vez va estar influenciado por factores socio-culturales, frente a estos cambios físicos, vasomotores, psicosociales y sexuales, dependen también de las características de la personalidad y sus vivencias acerca del climaterio, partiendo del hecho que es una etapa natural en la vida de la mujer y no es enfermedad, tiene una duración de 10 a 15 años tiempo en el cual ocurren cambios significativos en el organismo.

El climaterio es una etapa natural en la vida de la mujer, donde acontecen una serie de eventos que repercuten en su bienestar, este período generalmente duplica en tiempo a la niñez y la adolescencia; consiste en la transición de la etapa reproductiva a la no reproductiva debido a la disminución y el cese de la actividad ovárica y según se manifiesten los síntomas surge como crisis de la vida media que va a incidir en nivel de percepción del climaterio, que es el campo de investigación de este estudio, ya que se presentan sentimientos negativos e incertidumbre para enfrentar esta etapa, lo que conlleva al aumento de patologías porque no le da importancia a los síntomas que inicialmente presenta.

Entre las causas que originan la deficiente percepción del climaterio son: el bajo nivel de escolaridad, el desconocimiento del climaterio, insuficiente información recibida sobre el tema, poca comprensión del entorno familiar que pueden ahondar su crisis, las exigencias sexuales por parte de su pareja le crean conflictos en su diario vivir, las variables influencias hormonales con sus respectivos cambios físicos y funcionales influyen en la libido sexual; las falsas ideas y mitos que hacen que muchas mujeres tengan inseguridad.

Las fases del climaterio son: premenopausia es el tiempo que precede a la menopausia entre 3 a 5 años, cuando inician la caída de la función ovárica, la perimenopausia es el lapso de 1 a 2 años antes y después de la menopausia, tiempo en que los síntomas son notorios y el signo más evidente son los trastornos menstruales como la oligomenorrea y la amenorrea, la menopausia que es el tiempo transcurrido desde su F.U.M. hasta un año ininterrumpido de amenorrea, la posmenopausia comienza luego del año del F.U.M incluye los 7 a 10 años posteriores a la menopausia hasta el inicio de la vejez (Novak, 2012, pág. 1234). 
Menopausia proviene del griego meno $=$ mes y pause $=$ cese; es la interrupción permanente de la menstruación más de 12 meses consecutivos, en esta etapa se producen los cambios endocrinológicos, biológicos, psíquicos, y clínicos, la menopausia pude ocurrir a los 50 +/- 2 años de edad, puede presentarse de manera fisiológica, iatrogénica producida por extirpación quirúrgica de los ovarios o por causas tóxicas por tratamientos farmacológicos, quimioterapia o radioterapia (Williams, 2012, pág. 469). Según su aparición: es menopausia precoz cuando se presenta antes de los 40 años, menopausia adelantada entre los 40 a 45 años de edad, y la retrasada que se presenta después de los 55 años, en ciertos estudios a nivel de Latinoamérica ocurre alrededor de los 49 años.

En relación a la sintomatología que se presentan en el climaterio: está en relación con el déficit de estrógenos, infertilidad y síntomas de envejecimiento, según Williams los divide en síntomas a largo, mediano y corto plazo, los síntomas a corto plazo son: sangrados irregulares, los trastornos vasomotores: los sofocos, sudoraciones, y palpitaciones; los síntomas psíquicos son: cambios de humor, cefaleas, fatigas, depresión, descenso de la libido, perturbaciones del sueño, aumento de peso; los síntomas a mediano plazo son: modificaciones a nivel urogenital con sequedad vaginal, dispareunia, cistitis incontinencia e infecciones urinarias recurrentes, descenso y prolapsos, y los síntomas a largo plazo son: enfermedad cardiovascular, metabólicas, osteoporosis y a muy largo plazo: enfermedad de Alzheimer (Williams, 2012, pág. 507).

Los trastornos urogenitales y la atrofia urogenital según Testa son frecuente en el climaterio, el PH vaginal varía es ácido de 4,5 a PH 7 alcalino que permite el desarrollo de infecciones de origen exógenas o endógenas, el déficit de estrógeno produce también deficiente lubricación y elasticidad vaginal ocasionando dispareunia, en ocasiones puede haber micro traumatismos en la mucosa vaginal durante el coito o en el examen ginecológico por friabilidad de la mucosa originando micro-sangrado, puede presentarse prurito vulvar de variada intensidad acompañado de atrofia o distrofias genital en mujeres mayores (Testa, 2011, pág. 317). La dispareunia es una causa frecuente de la disminución del deseo sexual, puede presentarse 
cistitis, disuria e infecciones e incontinencia urinaria por atrofia el epitelio vesical y por la exposición la mucosa del meato urinario. Hay las modificaciones del suelo pélvico y genital por pérdida de elasticidad y sostén; los cambios que se observan en vulva son: perdida del vello púbico y se vuele canoso, los labios disminuyen el grosor y su elasticidad aparecen defectos de cierre del introito vaginal como son la atrofia y la distención ocasionando los descensos y prolapsos vaginales, (Usandizaga, 2010, pág. 530).

De acuerdo con las estadísticas de la Organización Mundial de la Salud (OMS) en pocos años se alcanzará la cifra mundial de 750 millones de mujeres posmenopáusicas (Búmel M., 2014), en los Estados Unidos de América, más de 30\% de la población femenina es posmenopáusica y en Europa, se estima que $12,3 \%$ de habitantes tiene más de 64 años; con el tiempo, este porcentaje deberá incrementarse. En Ecuador las expectativas de vida en el 2013 para la mujer fue de 79,40 años y para el año 2050 será de 83,5 años (Cepal, 2015).

En cuanto a la depresión y menopausia aproximadamente el $10 \%$ de las mujeres en el climaterio atraviesan por una depresión, lo cual provoca que la mujer experimente sentimientos de desgano, irritabilidad e inestabilidad emocional, disminución del apetito sexual (libido) y nerviosismo, estos estudios han demostrado que la depresión en las mujeres climatéricas estaba significativamente influenciada por la ocupación, el estatus económico, la armonía matrimonial y la misma menopausia, la irritabilidad está presente hasta en $70 \%$ de los casos; el 23\% se acompaña de síntoma depresivos, se asocian con insomnio, despertar temprano con incapacidad de volverse a dormir $(21 \%)$, apnea del sueño, y privación total del sueño. (Couto D,Nápoles,D, 2014).

La justificación de esta investigación es, porque existe desconocimiento del climaterio no se reconocen algunos síntomas y sus complicaciones afectan la salud física, mental, emocional y repercutiendo en el entorno familiar y social, por lo tanto es necesario plantear una estrategia que contribuya a la solución de este problema de salud reproductiva, que permitirá identificar al síndrome climatérico, favoreciendo a la 
mujer en la detección oportuna de enfermedades agregadas a través de pertinentes métodos de diagnósticos y recibir tratamientos adecuados lo que incidirá en la calidad de vida de las usuarias.

El objetivo general es elaborar un plan preventivo para evitar complicaciones del síndrome climaterio, a las usuarias del Centro Materno Infantil Francisco Jácome, y los objetivos específicos son: determinar los referentes teóricos generales sustantivos y empíricos sobre el climaterio y la percepción de las usuarias durante esta etapa, describir la metodología que analizará los factores sociales, educativos, culturales, biológicos, y psicológicos que influyen en la percepción del síndrome climatérico en mujeres de 40 a 65 años de edad, y elaborar un plan preventivo para evitar complicaciones del síndrome climatérico a las usuarias atendidas en el centro de salud.

\section{Metodología.}

En este proyecto de investigación es el estudio de caso y se emplea el método fenomenológico; que habla de la modificación de la conducta, de las actitudes, del sistema de creencias, de valores personales y sociales, de construcciones mentales y psicológicas sobre la salud y sobre las acciones individuales con la intención de capturar el fenómeno de estudio de manera holística, entenderlo, comprenderlo dentro de su contexto o enfatizar la comprensión del significado humano adscrito a un grupo de circunstancias o fenómeno. Y de las variantes de tipos de estudios de casos, es el de caso "típico", donde una persona puede representar a un grupo o comunidad, pueden estudiarse varias personas que tienen alguna característica en común, por lo que se espera cierta homogeneidad o coherencia en sus respuestas, para lo cual se recolectan datos a través de encuestas y entrevistas, se analizan, interpretan y validan.

La encuesta es un instrumento dentro del diseño de investigación descriptivo, en donde se busca recopilar datos por medio de un cuestionario previamente diseñado, sin modificar el entorno ni el fenómeno en donde se recoge la información y se realiza en menor tiempo, los datos se obtienen mediante un conjunto de preguntas normalizadas dirigidas a una muestra representativa o al conjunto total de la población 
de estudio, con el fin de conocer estados de opinión, ideas, características o hechos específicos. En este caso se emplea la encuesta con modalidad de pregunta cerrada y abierta y se las dividió en grupo según la dimensión a investigar constan: edad, nivel de instrucción, conocimiento del tema, estado civil, edad de la menopausia, percepciones de los cambios a nivel biológico, psicológico y sociocultural, se emplea la escala de Likert modificada a tres opciones para evitar mucha vacilación de las respuestas, y se realizó una encuesta al personal de salud en el área gineco-obstétrica con preguntas cortas y abiertas.

En este estudio se incluyó a 20 mujeres usuarias que acuden a la consulta externa del C.M.I. en edades comprendida de 40 a 60 años en quienes se realizó encuesta sobre la percepción de los síntomas climatéricos, para este estudio se consideró el rango de 46 a 50 años donde se encontró la mayor frecuencia de presentación de la menopausia y la edad promedio fue a los 48 años; comparando con un estudio que realizó Redlinc por Dr. Juan Blümel en año 2014 (Búmel M., 2014), sobre menopausia en relación a la población latinoamericana indica que la menopausia es alrededor de los 49 años lo que indica que coincide con la edad promedio que resultó de este estudio.

\section{Resultados.}

- En los referentes sustantivos de la percepción climatérica en usuarias del centro materno infantil Francisco Jácome se encontró que la mayoría no conoce que es el climaterio y da definiciones erradas, pero identifican los síntomas como sofocos, sudoración y cambios de carácter que se presentan de manera frecuente e intensidad variable. La edad promedio de la menopausia es a los 48 años, el cual no difiere de la estadística general que indica que su promedio de aparición a los $50+/-2$ años, la mayoría no relacionan los otros síntomas que se presentan con este proceso climatérico cual están pasando y desconocen las complicaciones de salud que se pueden presentar si no se realizan controles médicos.

- En el referente empírico para conocer realmente cuál es la percepción que tienen las mujeres sobre el climaterio se llevó a cabo el estudio a un grupo de usuarias que se atienden en la unidad de salud Francisco Jácome y los resultados fueron los siguientes: en relación a los datos demográficos como la 
edad la mayoría se encuentra entre las edades de 50 a 60 años, en relación al estado civil y ocupación se observa que la mayoría de las usuarias tiene una relación estable entre casadas y de unión libre; en cuanto a la ocupación casi los dos tercios son amas de casa; todas las usuarias tienen su residencia en el sector y pertenecen a ese distrito urbano, la raza predominante es la mestiza.

- En cuanto a la edad que tuvo su última menstruación se encontró, que más de la mitad de las usuarias estaban en el promedio de 46 a 50 años y de este grupo más de la mitad su último periodo menstrual fue a los 48 años. En la categoría educativa en lo referente a la escolaridad los resultados indican que la mitad tienen instrucción media, y el resto están con escolaridad primaria y sin instrucción. En lo que concierne al conocimiento del climaterio la gran mayoría no lo conoce y da definiciones erradas, solo menos de un quinto elige una definición acertada.

- En la categoría biológica se preguntó sobre la percepción de síntomas climatéricos, respondiendo en cuanto a los síntomas vasomotores la mayoría que los sofocos y sudoración está presente siempre y algunas veces. En lo referente a cefaleas que lo presentan más de la mitad entre siempre y algunas veces. Con respecto al cansancio y el dolor del cuerpo piernas y brazos se presentan en la mayoría de los casos. De los síntomas urogenitales: del total de encuestadas las tres cuartas partes indican que tienen resequedad vaginal. Mientras que la dispareunia, cistitis y leve incontinencia, contestan la mitad de los casos.

- En la categoría psicológica en lo referente a la percepción de emoción y afectividad, la irritabilidad y que se le olvidan las cosas son las alteraciones que en la mayoría han presentado, seguido por la depresión, insomnio, temor, disminución de concentración. En cuanto a las relaciones sexuales hay disminución de la libido en casi las tres cuartas parte del grupo de estudio y que evitan las relaciones sexuales están en la parte efectos de mala lubricación. En cuanto a que si su pareja se preocupa y la apoya en estas dificultades refieren que la mitad no las comprenden, y solo un cuarto de ellos si se preocupan. 
- Se realizó encuestas al profesional de salud a cargo de la atención a las usuarias en la consulta externa: Obstetrices y Ginecólogas, las preguntas están orientadas a conocer si las usuarias en etapa del climaterio acuden a la consulta externa por control específico y preventivo, manifestando más de la mitad de los profesionales que las usuarias no acuden de manera preventiva, y una tercera parte de ellos mencionan que las usuarias si acuden por algún síntoma del climaterio, pero la gran mayoría las usuarias no reconocen los síntomas ni lo relacionan con este periodo, un pequeño grupo de usuarias sí reconocen y relacionan los sofocos, irritabilidad, la amenorrea con esta etapa. Además todos los profesionales manifestaron que no hay un programa sobre el climaterio a seguir en el MSP. Y ellos mayoritariamente consideran que si es factible elaborar un plan de prevención de complicaciones sobre este tema, estando de acuerdo todos los profesionales que sí es, importante que la mujer tenga buena percepción del climaterio.

\section{Conclusiones.}

- Determinando los referentes teóricos sobre el climaterio y de la percepción de la usuaria en esta fase, se profundizó y actualizó conocimientos sobre el proceso biológico, psicológico y cognitivo que ocurre por la deficiencia hormonal, así como los diferentes tratamientos que se han desarrollado de acuerdo a las sintomatologías de las pacientes, la percepción que tiene las mujeres del climaterio varía según las vivencias y los factores socioculturales económico educativos que según ciertos autores ven a influir de forma negativa; así también

- siguen las investigaciones de los efectos por la deficiencia estrogénica en especial a nivel de las células nerviosas en el cerebro ya que este posee muchos receptores lo que permite el normal modulan el metabolismo de varios sistema de neurotransmisión, así como también los diversos tratamientos para los sofocos y osteoporosis. 
- En cuanto a la metodología del estudio de caso permitió investigar las categorías y las dimensiones a través de encuestas en relación a la sintomatología y la percepción que tienen las usuarias de este centro de salud, la mayoría de las usuarias presentaron trastornos somático-vegetativos como los sofocos, sudoración, irritabilidad, cambios de carácter que afectan mayormente sus actividades diarias, se evidenció la mayoría de usuarias están en edades de 50 a 60años que presentan trastornos urogenitales como sequedad vaginal y dispareunia. Por lo tanto fueron las más afectadas en su entorno familiar en especial, ya que se generó conflictos en relación de pareja lo que afectó el buen desenvolvimiento de la vida familiar. La mayoría tienen desconocimiento de las complicaciones eso les genera mucho temor hace que tengan una mala percepción y actitud del climaterio

- Ante esta problemática estudiada es útil elaborar un plan de preventivo para mejorar la percepción del climaterio, en especial antes de presentarse el cuadro para que las mujeres sepan reconocer cuándo es fisiológico, deben recibir orientaciones sobre cómo manejar la situación, que también reconozcan cuando es una complicación, y acudan con prontitud a la unidad de salud para recibir tratamiento adecuado en el Centro Materno Infantil Francisco Jácome.

\section{Recomendaciones.}

- Seguir con la difusión del tema climaterio de manera continuada con su diversa sintomatología

- Sugerir realizar réplicas del tema climaterio con investigación cualitativa sobre percepción del climaterio en otras unidades de salud para que aporten nuevos datos de la situación real de las mujeres en otras zonas.

- Fortalecer la investigación sobre el climaterio con otros puntos importantes a nivel psicológico y de relación de pareja a profundidad ya que se evidenció la presencia conflictos familiares en este grupo vulnerable de mujeres. 
- Fomentar eficientemente la prevención de los factores de riesgo de las complicaciones durante el climaterio ya que es un problema de salud en el hogar y de salud pública.

- Realizar continuamente difusión sobre cuidados y autocuidados orientado a mejorar la calidad y expectativas de vida en las mujeres, ya que el tratar las complicaciones por enfermedad generan costos a la familia y al estado.

- Brindar una atención médica específica, indicar la importancia de la consulta temprana y del diagnóstico oportuno de posibles complicaciones y su tratamiento.

- Involucrar a todo el personal médico del establecimiento en captar a las mujeres que se encuentran en esta etapa, brindar información precisa y actual acerca del climaterio, en la consulta externa formar grupos afines para realizar educación, asesorías, conferencias, talleres interactivos sobre del climaterio.

\section{Bibliografía.}

1. Novak JS. Ginecologia de Novak. In Novak B. Ginecologia de Novak. Philadelphia: Lippincott Williams \& Wilkins; 2012. p. 1234,1238,1239,1240, 1244.

2. Williams. Ginecologia. In C.Gary. Ginecologia d Williams. México: Mac GGraw-Hill; 2012. p. $469,470,484,473,474,479.483,507$.

3. Testa R. Ginecologia. In Testa R. fundamentos practicos de ginecología. Buenos Aires: Médica Panamericana; 2011. p. 317, 318.319.

4. Usandizaga. In Usandizaga J D1F. Tratado de obstetricia y ginecología. Madrid: McGraw-Hill; 2010. p. 530,533.

5. Búmel M. JE. REDLINC Red latinoamericana de investigación del climaterio. Revista chilena menopausia fact. riesgos. 2014;: p. varias.
6. Cepal.
www.cepal.org/.
[Online];
2015.
Available
from: (http://www.cepal.org/es/publicaciones/38713-.

7. Couto D,Nápoles,D. aspectos psicologicos del climaterio Stgo Cuba. [Online].; 2014. 
Vol. 5, núm.1. ene., 2019, pp. 722-735

Percepción del climaterio en pacientes del centro de salud materno infantil "Francisco Jácome”, plan de prevención

8. Testa R. ginecologia. In Testa R. fundamentos practicos de Ginecologia. Buenos Aires: Médica Panamericana; 2011. p. 314,315, 329,334.335.

9. Hernandez,Fernandez,Baptista. investigación cualitativa. In.; 2003. 